\title{
Array comparative genomic hybridization analysis of olfactory neuroblastoma
}

\author{
Mohamed Guled ${ }^{1}$, Samuel Myllykangas ${ }^{1}$, Henry F Frierson $\mathrm{Jr}^{2}$, Stacey E Mills ${ }^{2}$, \\ Sakari Knuutila ${ }^{1}$ and Edward B Stelow ${ }^{2}$ \\ ${ }^{1}$ Department of Pathology, Haartman Institute and HUSLAB, University of Helsinki and Helsinki University \\ Central Hospital, Helsinki, Finland and ${ }^{2}$ Department of Pathology, University of Virginia Health System, \\ Charlottesville, VA, USA
}

\begin{abstract}
Olfactory neuroblastoma is an unusual neuroectodermal malignancy, which is thought to arise at the olfactory membrane of the sinonasal tract. Due to its rarity, little is understood regarding its molecular and cytogenetic abnormalities. The aim of the current study is to identify specific DNA copy number changes in olfactory neuroblastoma. Thirteen dissected tissue samples were analyzed using array comparative genomic hybridization. Our results show that gene copy number profiles of olfactory neuroblastoma samples are complex. The most frequent changes included gains at 7q11.22-q21.11, 9p13.3, 13q, 20p/q, and Xp/q, and losses at 2q31.1, 2q33.3, 2q37.1, 6q16.3, 6q21.33, 6q22.1, 22q11.23, 22q12.1, and Xp/q. Gains were more frequent than losses, and high-stage tumors showed more alterations than low-stage olfactory neuroblastoma. Frequent changes in high-stage tumors were gains at 13q14.2-q14.3, 13q31.1, and 20q11.21-q11.23, and loss of Xp21.1 (in $66 \%$ of cases). Gains at 5q35, 13q, and 20q, and losses at 2q31.1, 2q33.3, and 6q16-q22, were present in $50 \%$ of cases. The identified regions of gene copy number change have been implicated in a variety of tumors, especially carcinomas. In addition, our results indicate that gains in $20 \mathrm{q}$ and $13 \mathrm{q}$ may be important in the progression of this cancer, and that these regions possibly harbor genes with functional relevance in olfactory neuroblastoma.
\end{abstract}

Modern Pathology (2008) 21, 770-778; doi:10.1038/modpathol.2008.57; published online 11 April 2008

Keywords: olfactory neuroblastoma; array CGH; esthesioneuroblastoma; cytogenetics

Olfactory neuroblastoma is rare, with an estimated incidence of 0.4 per million people per year. It accounts for approximately $2-3 \%$ of sinonasal tract tumors. ${ }^{1}$ Olfactory neuroblastoma is a malignant neuroectodermal tumor, which is believed to originate at the olfactory membrane of the sinonasal tract. ${ }^{1}$ Symptoms include unilateral nasal obstruction and epistaxis. The Kadish system classifies cases of olfactory neuroblastoma into three stages. Stage 1 consists of tumors confined to the nasal cavity; stage 2 tumors extend to the paranasal sinuses; and stage 3 neoplasms extend beyond the sinonasal cavities (some have added a stage 4 for those tumors with metastases). Patients with high-

Correspondence: Dr EB Stelow, MD, Department of Pathology, University of Virginia Health Sciences, Box 800214, Jefferson Park Avenue, Charlottesville, VA 22908, USA.

E-mail: edstelow@yahoo.com and

Dr S Knuutila, PhD, Department of Pathology, Haartman Institute and HUSLAB, PO Box 21 (Haartmaninkatu 3), University of Helsinki, FI-00014 Helsinki, Finland.

E-mail: Sakari.Knuutila@helsinki.fi

Received 24 November 2007; revised 02 March 2008; accepted 10 March 2008; published online 11 April 2008 stage tumors have a worse prognosis compared with those with low-stage tumors, as 5-year survival rates are 40 and $80 \%$, respectively. ${ }^{2}$

Cytogenetic data for olfactory neuroblastoma are limited. The most recently published study was carried out by Holland et al, ${ }^{3}$ who performed cytogenetic characterization of one case using trypsin Giemsa staining (GTG banding), multicolor fluorescence in situ hybridization (M-FISH), and single-nucleotide polymorphism karyotyping. They reported numerous chromosomal aberrations predominantly involving chromosomes 2q, 5, 6q, 17, 19, 21q, and 22, as well as trisomy 8 . Bockmüehl et $a l^{4}$ applied conventional comparative genomic hybridization (CGH) to 22 olfactory neuroblastomas and reported frequent deletions of $1 p, 3 p / q, 9 p$, and $10 \mathrm{p} / \mathrm{q}$, and amplifications of $17 \mathrm{q}, 17 \mathrm{p} 13,20 \mathrm{p}$, and 22q. They also noted a specific deletion on chromosome 11 and gain on chromosome 1p, which were associated with metastasis and a worse prognosis. Three olfactory neuroblastomas were studied by Riazimand et $a l^{5}$ using conventional CGH, and amplification of whole chromosome 19, partial gains of $1 p, 8 q, 15 q$, and $22 q$, and deletions of $4 q$ and $6 p$ 
were detected. Szymas et $a l^{6}$ studied a single olfactory neuroblastoma and found gains of whole chromosomes 4, 8, 11, and 14, partial gains of $1 \mathrm{q}$ and $17 \mathrm{q}$, partial deletions of $5 \mathrm{q}$ and $17 \mathrm{q}$, and whole chromosome losses of 16, 18, 19, and X. In our study, we applied for the first time an oligonucleotide-based array CGH (aCGH) to identify the most frequently occurring DNA copy number changes in 13 cases of olfactory neuroblastoma. We identified novel chromosomal regions that were frequently altered in addition to previously reported abnormal regions.

\section{Materials and methods}

\section{Tumor Samples}

The Surgical Pathology Database (Department of Pathology at the University of Virginia, Charlottesville, VA, USA) was searched for resected cases of olfactory neuroblastoma. Slides from formalin-fixed, paraffin-embedded tissues were reviewed, and 15 cases were selected based on the availability of abundant and well-preserved neoplastic tissue. Only cases with conventional histology, as described by Mills and Frierson, ${ }^{7}$ were chosen (Figure 1). All cases exhibited a typical immunophenotype and were immunoreactive with antibodies to synaptophysin and not with antibodies to keratins. Areas representing cancer tissue were dissected from paraffin-embedded tumors using conventional light microscopy and scalpel blades. All 15 samples submitted for analysis were composed of at least $50 \%$ tumor cells. Each of six samples was classified as Kadish stage 2 and 3 (Figure 1), whereas information regarding the stage was not available for three tumors. Patients' age ranged from 27 to 62 years. The clinicopathological data for the patients and samples are summarized in Table 1.

\section{DNA Extraction and Digestion}

Total DNA was extracted using QIAamp DNA mini kit, according to the manufacturer's instructions (Qiagen, Hilden, Germany). DNA concentrations of the extracted samples were determined using the NanoDrop ND-1000 spectrophotometer (Nanodrop technologies, Wilmington, DE, USA) at $260 / 280 \mathrm{~nm}$. The integrity of the extracted DNA was verified by agarose gel electrophoresis (data not shown). A $1.5 \mu \mathrm{g}$ quantity of genomic DNA from test and reference samples was digested using Alu1 and Rsa1 restriction enzymes (Promega, Madison, WI, USA) for $2 \mathrm{~h}$ at $37^{\circ} \mathrm{C}$. To inactivate the enzymes, the samples were incubated at $65^{\circ} \mathrm{C}$ for $20 \mathrm{~min}$.

\section{Array CGH Experiments}

Array CGH was performed for 13 samples that had good quality DNA with sufficient yields. For

Table 1 Clinicopathological data for the samples

\begin{tabular}{|c|c|c|c|c|c|}
\hline Sample & $\begin{array}{l}\text { Sex/ } \\
\text { age }\end{array}$ & $\begin{array}{l}\text { Dead/ } \\
\text { alive }\end{array}$ & $\begin{array}{c}\text { Disease } \\
\text { status }\end{array}$ & $\begin{array}{c}\text { Time } \\
\text { (months) }\end{array}$ & $\begin{array}{c}\text { Stage } \\
\text { (Kadish) }\end{array}$ \\
\hline 1 & $\mathrm{~m} / 50$ & $\mathrm{D}$ & WD & 156 & 2 \\
\hline 2 & $\mathrm{~m} / 49$ & A & NED & 192 & UNK \\
\hline 3 & $\mathrm{~m} / 53$ & D & WD & 20 & 3 \\
\hline 4 & $\mathrm{f} / 38$ & A & UNK & 136 & UNK \\
\hline 5 & $\mathrm{f} / 40$ & A & UNK & 149 & 2 \\
\hline 6 & $\mathrm{f} / 27$ & A & NED & 119 & 3 \\
\hline 7 & $f / 61$ & A & NED & 106 & 3 \\
\hline 8 & $\mathrm{~m} / 27$ & A & NED & 59 & 2 \\
\hline 9 & $\mathrm{~m} / 40$ & A & NED & 57 & 3 \\
\hline 12 & $\mathrm{f} / 62$ & D & WD & 35 & 3 \\
\hline 13 & $\mathrm{~m} / 41$ & UNK & UNK & UNK & 3 \\
\hline 14 & $\mathrm{~m} / 44$ & A & NED & 24 & UNK \\
\hline 15 & $\mathrm{~m} / 60$ & A & NED & 6 & 2 \\
\hline
\end{tabular}

Abbreviations: A, alive; D, dead; NED, no evidence of disease; UNK, unknown; WD, with disease.
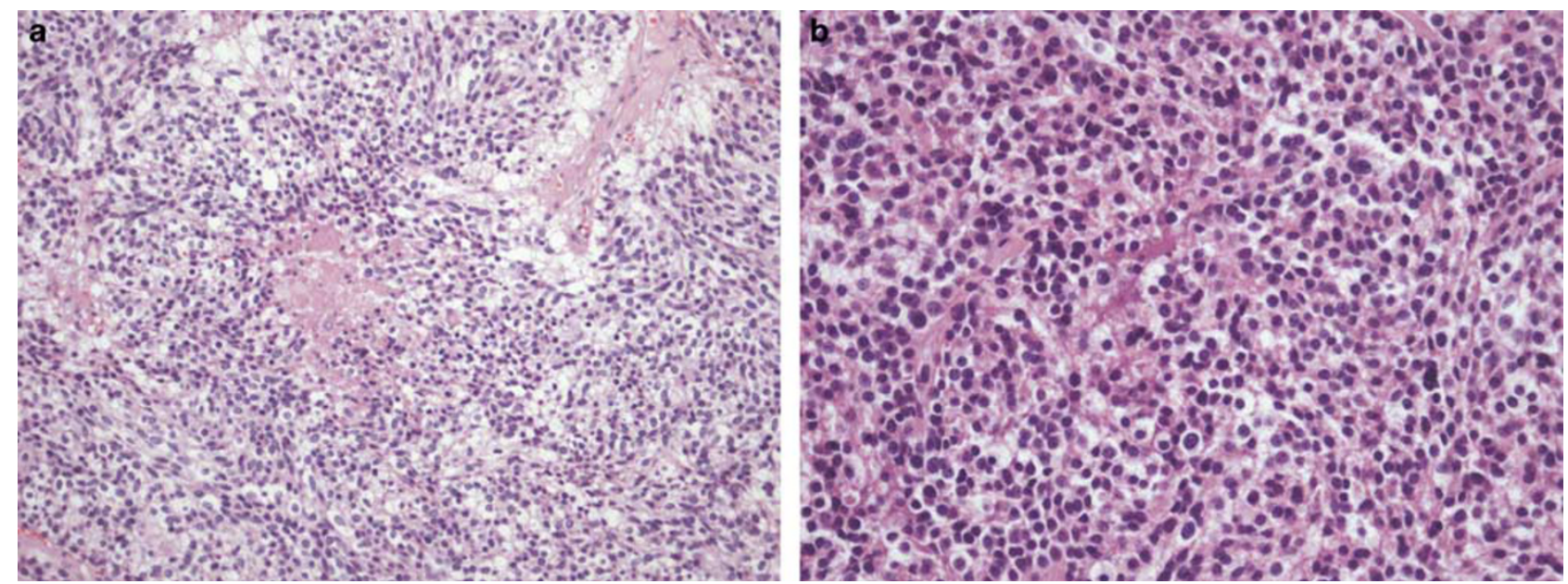

Figure 1 Standard H\&E sections of conventional olfactory neuroblastoma: (a) sample $11(100 \times)$, (b) sample $12(200 \times)$. 
reference, pooled DNA extracted from buffy coat fractions of whole blood obtained from sex-matched, healthy individuals provided by the Finnish Red Cross was used. The Agilent genomic DNA labeling kit PLUS (Agilent Technologies, Santa Clara, CA, USA) was used to label $1.5 \mu \mathrm{g}$ of digested genomic DNA. The DNA of olfactory neuroblastoma samples and the reference DNA were labeled with cyanine 5dUTP and cyanine 3-dUTP fluorochromes, respectively, for $2 \mathrm{~h}$ at $37^{\circ} \mathrm{C}$. For inactivation of the enzymes, the samples were incubated at $65^{\circ} \mathrm{C}$ for 10 min. Human cot-1 DNA (Invitrogen, Carlsbad, CA, USA) was added to the reactions for $3 \mathrm{~min}$ at $95^{\circ} \mathrm{C}$ and then for $30 \mathrm{~min}$ at $37^{\circ} \mathrm{C}$. Prior to hybridization experiments, labeled DNA was cleaned according to Agilent's protocol. Labeled sample and reference DNA were hybridized on Agilent's $4 \times 44 \mathrm{~K}$ CGH microarray (product number G4426A and G4426B, Agilent Technologies) for $24 \mathrm{~h}$ at $65^{\circ} \mathrm{C}$.

Prior to scanning, microarray slides were washed according to the manufacturer's instructions. The microarray slides were scanned using DNA Microarray Scanner and the scanned images were analyzed using the Feature Extraction Software (Agilent Technologies). Data were imported to the CGH Analytics Software 3.4 for analysis of individual cases. $Z$-score algorithm with threshold at 3.5 was used in the identification of copy number changes. To obtain a global view of the copy number alteration frequencies in olfactory neuroblastoma, we also analyzed the data using GeneSpring (Agilent Technologies) and CGH Explorer software 3.1. ${ }^{8}$ The raw data from the Feature Extraction software were imported into the GeneSpring software and normalized using the Feature Extraction data import plugin (Agilent Technologies). Outlier probes marked by Feature Extraction were ignored in normalization. Sample spot intensities were divided by control channel intensities, and microarrays were normalized to the 50th percentile and probes to the median value. Outlier probes were filtered from further analysis. Normalized and filtered data were imported into the CGH Explorer software. Copy number aberrations were identified using Piecewise Constant Fit (PCF) algorithm. Default parameters were applied to detect long copy number aberrations. The significance threshold was set at 0.2.

\section{Results}

\section{Gene Copy Number Profiling of Olfactory Neuroblastoma}

Array CGH was applied to study gene copy number alterations in 13 olfactory neuroblastoma samples. The CGH Explorer software was used to analyze global frequencies of copy number change (Figure 2). A total of $15.74 \%$ of the probes were found aberrant when a threshold of 0.2 was used in the PCF analysis. DNA copy number gains $(8.37 \%)$ were slightly more frequent than losses $(7.36 \%)$. In addition, the CGH analytics software was used to determine copy number aberrations in individual samples. Copy number aberrations for each case are summarized in Table 2. Changes in the copy number of entire chromosomes (aneuploidy) or chromosome

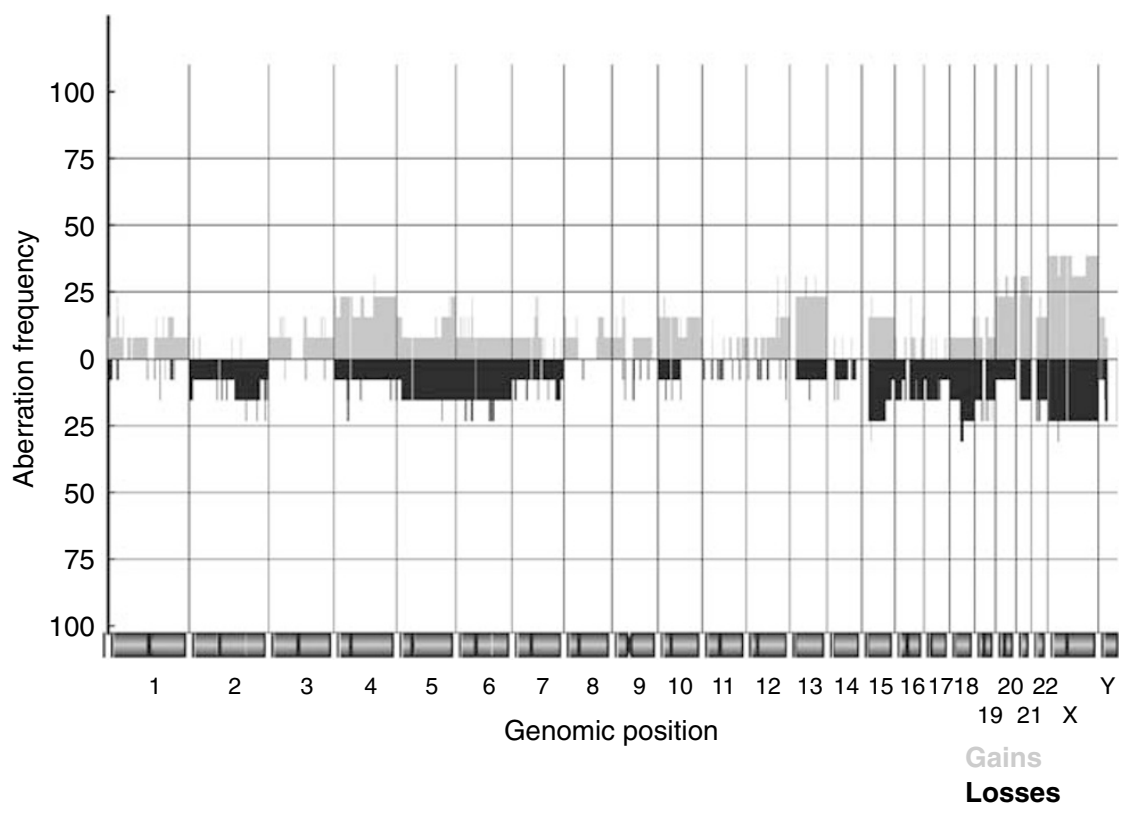

Figure 2 Frequency profile of copy number aberrations in 13 olfactory neuroblastoma samples. CGH Explorer software and Piecewise Constant Fit algorithm were used to determine copy number aberrations in olfactory neuroblastoma samples. The chromosomal alterations are shown in each probe position as incidence bar. Gains of genomic material are indicated in gray on the upper side of the middle line (at 0). Losses are indicated in black on the bottom side of the middle line. Genomic positions of the aCGH probes are marked on the $x$ axis. 
Table 2 aCGH results for 13 ONB samples

\begin{tabular}{|c|c|c|}
\hline ONB sample & Stage & Tumor DNA copy number changes \\
\hline 1 & 2 & $\begin{array}{l}+1 \mathrm{p} 31.2,+1 \mathrm{p} 31.1,+1 \mathrm{p} 35.3,+1 \mathrm{q} 31.1 \mathrm{q} 31.3,+2 \mathrm{p} 24.3,-2 \mathrm{p} 25.1 \mathrm{p} 25.3,-2 \mathrm{q} 36.3 \mathrm{q} 37.3,+3 \mathrm{p} 13.12 \mathrm{p} 13.31,+4 \mathrm{p},+4 \mathrm{q}, \\
+5 \mathrm{q} 23.1,+8 \mathrm{q} 22.3 \mathrm{q} 24.12,-9 \mathrm{p} 21.1,-10 \mathrm{p} 12.31,-11 \mathrm{q} 14.1 \mathrm{q} 14.3,-12 \mathrm{q} 12,-16 \mathrm{q} 13,-19 \mathrm{p},-19 \mathrm{q},-22 \mathrm{q}\end{array}$ \\
\hline 2 & UNK & $\begin{array}{l}+1 \mathrm{p} 21.1 \mathrm{p} 21.2,+1 \mathrm{q} 31.3 \mathrm{p} 32.1,-2 \mathrm{q} 37.1,+3 \mathrm{q} 25.33,+4 \mathrm{p} 16.2 \mathrm{p} 16.3,+4 \mathrm{p} 12 \mathrm{p} 15.31,+4 \mathrm{q},+6 \mathrm{p} 21.2,+9 \mathrm{p} 23, \\
+12 \mathrm{p} 11.21 \mathrm{p} 12.1,+12 \mathrm{q} 23,+12 \mathrm{q} 24.31,-12 \mathrm{q} 12,+16 \mathrm{q} 23.2 \mathrm{q} 24.2,+17 \mathrm{q} 11.2,-18 \mathrm{p},-18 \mathrm{q},-19 \mathrm{p},-19 \mathrm{q},+22 \mathrm{q},+\mathrm{Xp}, \\
+\mathrm{Xq12}\end{array}$ \\
\hline 3 & 3 & $\begin{array}{l}-2 q 37.2,+3 q 21.32 p 21.33,+3 p 21.31,+3 q 25.33,+4 p,+4 q 12,+4 q 35.2,-5 p,-5 q,+6 p 22.3,-6 q 21,-7 p \\
-7 q 11.21 q 21.2,-7 q 33 q 35,+8 p 23.2 p 23.3,+8 p 12,+10 p 11.21 p 15.3,+10 q 11.21 q 21.1,+10 q 23.1 p 26.2 \\
+12 q 21.33 q 24.33,+13 q 31.1,+13 q 34,-14 q 32.33,-15 q 11.2 q 13.1,+16 q 24.2,-16 p 12.3 p 13.2,-16 q 22.1 q 24.2 \\
-17 p,-17 q 11.2 q 12.31,-18 p,-18 q,+19 q 13.12,+20 q 11.2111 .25,+20 q 13.32 q 13.33,-22 q\end{array}$ \\
\hline 4 & UNK & $\begin{array}{l}+1 q 44,-1 q 32.1,+2 p 25.3,+3 q 26.2,-3 q 26.2,-4 q 35.2,+5 q 34,-5 q 31.2,+6 p 12.3,-8 q 12.1,-9 p 13.1,-9 q 12 q 13, \\
+11 p 13,-11 p 14.1,-11 q 21,+12 q 23.1,+14 q 23.3,-14 q 11.2,-15 q 11.2 q 25.3,+16 p 13.3,+16 p 11.2,-16 p 13.3 \\
-16 p 12.1,+21 q 21.1 q 21.3\end{array}$ \\
\hline 5 & 2 & $\begin{array}{l}+1 \mathrm{p} 35.2 p 35.3,+1 p 36.11,-1 \mathrm{p} 36.11,+3 \mathrm{p} 22.3,+3 q 26.2,-3 \mathrm{p} 21.31,-3 \mathrm{q} 26.2,+4 \mathrm{q} 27,+9 \mathrm{p} 24.3,+11 \mathrm{p} 15.5 \\
+11 \mathrm{q} 13.1,+12 \mathrm{q} 24.31,-12 \mathrm{q} 14.3,-18 \mathrm{q} 12.2 \mathrm{q} 12.3,+13 \mathrm{q} 34,+19 \mathrm{p} 13.2\end{array}$ \\
\hline 6 & 3 & $\begin{array}{l}+1 \mathrm{p} 34.1 \mathrm{p} 36.33,+1 \mathrm{p} 31.2 \mathrm{p} 32.1,+1 \mathrm{p} 21.2 \mathrm{p} 22.3,+1 \mathrm{q} 21.1 \mathrm{q} 21.3,+1 \mathrm{q} 22 \mathrm{q} 24,+2 \mathrm{q} 14.1,+2 \mathrm{q} 21.1,-2 \mathrm{q} 31.1 \\
+3 \mathrm{p} 14.1 \mathrm{p} 26.3,+3 \mathrm{q} 13.11 \mathrm{q} 29,+4 \mathrm{q} 22.1 \text { to q21.22, -5p11p15.1, -5q, -6q12.3, -6q16.3, -6q21q22.1, +7q22.1, } \\
+8 \mathrm{p},+8 \mathrm{q} 22.1 \mathrm{q} 24.3,+9 \mathrm{p} 21.1,+9 \mathrm{p} 15.2 \mathrm{p} 13.3,-10 \mathrm{q} 21.3,-11 \mathrm{p} 15.4,-11 \mathrm{p} 11.12 \mathrm{p} 11.2,-11 \mathrm{q} 11 \mathrm{q} 12.2,-11 \mathrm{q} 13, \\
\mathrm{q} 23-2,+12 \mathrm{p} 13.31 \mathrm{p} 13.33,+12 \mathrm{q} 12 \mathrm{q} 13.12,+12 \mathrm{q} 13.13 \mathrm{q} 14.1,+12 \mathrm{q} 14.2 \mathrm{q} 23.1,+12 \mathrm{q} 23.3 \mathrm{q} 24.33,+13 \mathrm{q} 14.2 \mathrm{q} 14.3 \\
-14 \mathrm{q} 12 \mathrm{q} 23.3,-14 \mathrm{q} 31.1 \mathrm{q} 31.3,-16 \mathrm{p} 11.2,-16 \mathrm{q} 11.2,+17 \mathrm{q} 21.2,+17 \mathrm{q} 24.2,+17 \mathrm{q} 25.1,-17 \mathrm{q} 24.3,-18 \mathrm{q} 12.2 \mathrm{q} 23 \\
-19 \mathrm{p} 12,-19 \mathrm{q} 12,-20 \mathrm{p},-20 \mathrm{q},-22 \mathrm{q} 11.21,-\mathrm{Xp},-\mathrm{Xq}\end{array}$ \\
\hline 7 & 3 & $\begin{array}{l}-1 \mathrm{q} 31.2,-6 \mathrm{p},-6 \mathrm{q},-7 \mathrm{q} 31.1,-10 \mathrm{q} 11.21,+12 \mathrm{p} 13.31,-12 \mathrm{q} 24.23 \mathrm{q} 24.31,-12 \mathrm{q} 24.31,+13 \mathrm{q},+14 \mathrm{q} 24.3,-15 \mathrm{q} \\
-16 \mathrm{q} 12.1 \mathrm{q} 21,-16 \mathrm{q} 24.2,+17 \mathrm{p},+17 \mathrm{q},-19 \mathrm{q} 13.32,+21 \mathrm{q},-22 \mathrm{q} 11.23,-\mathrm{Xp},-\mathrm{Xq}\end{array}$ \\
\hline 8 & 2 & $\begin{array}{l}-1 \mathrm{p} 36.22 \mathrm{p} 36.33,-4 \mathrm{p},-4 \mathrm{q},-5 \mathrm{p} 15.33,+6 \mathrm{p} 22.1,+6 \mathrm{p} 12.3,-6 \mathrm{p} 22.1,-7 \mathrm{p} 12.3 \mathrm{p} 13,+9,+9 \mathrm{q} 21.11 \mathrm{q} 32.2,+9 \mathrm{q} 33.3, \\
+10 \mathrm{p},+10 \mathrm{q} 11.21 \mathrm{q} 23.1,+10 \mathrm{q} 23.3210 \mathrm{q} 26.3,+11 \mathrm{p} 13,-12 \mathrm{q} 24.31,-13 \mathrm{q},-14 \mathrm{q} 12,-21 \mathrm{q},-22 \mathrm{q} 12.11, \\
+\mathrm{Xp} 21.2 \mathrm{p} 22.33,+\mathrm{Xp} 11.22 \mathrm{p} 11.4,+\mathrm{Xq}\end{array}$ \\
\hline 9 & 3 & $\begin{array}{l}+1 \mathrm{p} 36.33,+1 \mathrm{p} 36.31,+1 \mathrm{p} 33,-1 \mathrm{q} 23.3,-1 \mathrm{q} 25.2,-2 \mathrm{p} 11.2,-2 \mathrm{q} 11.2,-2 \mathrm{q} 22.1 \mathrm{q} 35,-4 \mathrm{p} 13 \mathrm{p} 14,-4 \mathrm{q} 34.1 \\
+5 \mathrm{p} 15.2 \mathrm{p} 15.33,+5 \mathrm{q} 35.1 \mathrm{q} 35.3,-6 \mathrm{p},-6 \mathrm{q},+7 \mathrm{p},+7 \mathrm{q} 11.2 \mathrm{q} 21.2,-7 \mathrm{q} 21.3 \mathrm{q} 36.3,+9 \mathrm{p} 13.1,+9 \mathrm{q} 12 \mathrm{q} 13, \\
+12 \mathrm{p} 13.31 \mathrm{p} 13.33,+12 \mathrm{q} 24.33,-12 \mathrm{q} 23.3,+13 \mathrm{q},+14 \mathrm{q} 12,+15 \mathrm{q} 13.3,-15 \mathrm{q} 14 \mathrm{q} 24.1,-15 \mathrm{q} 14 \mathrm{q} 26.3,-16 \mathrm{p},-16 \mathrm{q}\end{array}$ \\
\hline 12 & 3 & $\begin{array}{l}-1 \mathrm{q} 23.3,-2 \mathrm{p},-2 \mathrm{q},-3 \mathrm{q} 13.2,+5 \mathrm{p},+5 \mathrm{q},+6 \mathrm{p},+6 \mathrm{q},+7 \mathrm{q} 36.1,+7 \mathrm{q} 36.3,-9 \mathrm{p} 13.1,-9 \mathrm{q} 13,-10 \mathrm{p},-10 \mathrm{q} 11.21 \mathrm{q} 21.3, \\
+11 \mathrm{q} 23.3,-12 \mathrm{p} 13.33,+13 \mathrm{q},-14 \mathrm{q} 13.2,-14 \mathrm{q} 24.3,+15 \mathrm{q} 11.2 \mathrm{q} 22.31,+15 \mathrm{q} 22.32 \mathrm{q} 26.33,+16 \mathrm{q} 12.1 \mathrm{q} 21,-17 \mathrm{p}, \\
-17 \mathrm{q}, 18 \mathrm{q} 25,+19 \mathrm{p} 13.2,+19 \mathrm{q} 12 \mathrm{q} 13.11,+20 \mathrm{p},+20 \mathrm{q},-21 \mathrm{q},-\mathrm{Xp},-\mathrm{Xq}\end{array}$ \\
\hline 13 & 3 & $\begin{array}{l}+1 \mathrm{q} 21.2,-2 \mathrm{q} 33.3,+5 \mathrm{q} 31.2 \mathrm{q} 35.3,+7 \mathrm{q} 11.23,+7 \mathrm{q} 32.1 \mathrm{q} 32.2,-7 \mathrm{p} 21.3,+9 \mathrm{p} 13.3,-9 \mathrm{q} 21.33,-10 \mathrm{p} 12.1 \mathrm{p} 12.2, \\
+11 \mathrm{q} 12.2,+11 \mathrm{q} 23.1 \mathrm{q} 23.2,-11 \mathrm{q} 23.2 \mathrm{q} 23.3,+14 \mathrm{q} 11.2,-17 \mathrm{q} 21.33 \mathrm{q} 22,+19 \mathrm{q} 13.33,+19 \mathrm{q} 13.43,+20 \mathrm{p},+20 \mathrm{q},+X p \\
+20 \mathrm{q}\end{array}$ \\
\hline 14 & UNK & $+1 \mathrm{p} 36.11,-4 \mathrm{q} 21.21,+2 \mathrm{p} 22.3 \mathrm{p} 23.1,+2 \mathrm{q} 24.1,-12 \mathrm{q} 13.2,-12 \mathrm{q} 14.3 \mathrm{q} 15,+16 \mathrm{q} 12.1,+19 \mathrm{p} 13.2,+22 \mathrm{q} 12.1$ \\
\hline 15 & 2 & $\begin{array}{l}-1 \mathrm{p} 35.2 \mathrm{p} 35.3,-1 \mathrm{p} 11.2 \mathrm{p} 12,-1 \mathrm{q} 21.1,-1 \mathrm{q} 31.3 \mathrm{q} 32.1,-4 \mathrm{p} 12 \mathrm{p} 13,-6 \mathrm{p} 21.33,+7 \mathrm{q} 11.22 \mathrm{q} 21.11,-9 \mathrm{q} 31.3 \\
-13 \mathrm{q} 21.2,-17 \mathrm{p} 13.1,+21 \mathrm{q} 22.12 \mathrm{q} 22.3,+22 \mathrm{q},+\mathrm{Xp},+\mathrm{Xq}\end{array}$ \\
\hline
\end{tabular}

arms represented $16 \%$ of all alterations; interestingly, the most altered whole chromosomes were 20, 21,22 , and $X$, and almost $70 \%$ of the aberrations of these were whole chromosome losses or gains.

To obtain a more detailed overview of the most frequently occurring alterations, only those alterations shared by at least $20 \%$ of the cases were recorded. This approach identified an equal number of gained and lost chromosomal regions $(n=21)$. Tables 3 and 4 show the size of the altered areas and whether they have been reported in previous copy number change studies for olfactory neuroblastoma. The tables also include suggested target genes that are located at the sites of alteration and that may play a role in tumorigenesis.

\section{Specific Copy Number Alterations Were Identified in Cases of Olfactory Neuroblastoma of Different Stage}

A stage 3 olfactory neuroblastoma showed the most DNA copy number changes, with $59 \%$ of all alterations. Also, most aneuploidy occurred in high-stage tumors; $70 \%$ of whole-chromosome aberrations were identified in these tumors. For stage 3 tumors, the mean DNA copy number change per case was 28.5, whereas stage 2 olfactory neuroblastomas had a mean number of 17 changes per tumor. However, only 4 out of 6 stage 2 cases contained sufficient good quality DNA to perform aCGH.

To further characterize the genomic changes that occurred in a later-stage olfactory neuroblastoma, 
Table 3 DNA copy number gains in at least $20 \%$ of the cases detected by aCGH

\begin{tabular}{|c|c|c|c|c|c|c|c|c|c|}
\hline $\begin{array}{l}\text { Chromosome } \\
\text { location }\end{array}$ & Start (kb) & Stop (kb) & $\begin{array}{c}\text { Size of } \\
\text { area }(\mathrm{kb})\end{array}$ & $\begin{array}{c}\text { No. of genes in } \\
\text { the area }\end{array}$ & $\begin{array}{l}\text { Suggested } \\
\text { target genes }\end{array}$ & $\begin{array}{l}\text { Szymas et al } \\
\text { (1997) }\end{array}$ & $\begin{array}{l}\text { Riazimand } \\
\text { et al (2002) }\end{array}$ & $\begin{array}{l}\text { Bockmühl et } \\
\text { al (2004) }\end{array}$ & $\begin{array}{l}\text { Holland et al } \\
\text { (2007) }\end{array}$ \\
\hline 1p36.31 & 6220 & 6385 & 165 & 4 & & & 1p32-pter & & \\
\hline 1p35.3 & 28196 & 28354 & 158 & 3 & & & & & \\
\hline $1 \mathrm{p} 35.3$ & 28625 & 28689 & 64 & 1 & & & & & \\
\hline 4p16.2-p16.3 & 1 & 6116 & 6116 & 94 & & 4 & & & \\
\hline 4p12-p15.31 & 19927 & 48749 & 28822 & 132 & & & & & \\
\hline $4 \mathrm{q} 12$ & 52152 & 56155 & 4003 & 27 & & & & & \\
\hline $4 q 21.22-q 22.1$ & 82376 & 90382 & 8006 & 71 & & & & & \\
\hline $4 q 27-q 35.2$ & 119605 & 188460 & 68855 & 349 & & & & & \\
\hline $4 q 27-q 35.2^{\mathrm{a}}$ & 123408 & 124209 & 801 & 38 & & & & & \\
\hline $5 q 34$ & 160870 & 161694 & 824 & 6 & & & & & \\
\hline $5 q 35.1-q 35.3$ & 167872 & 180708 & 12836 & 71 & & & & & \\
\hline 6p12.3 & 48645 & 1446 & 1446 & 12 & & & & & \\
\hline $7 q 11.23$ & 72440 & 73209 & 769 & 16 & $\begin{array}{l}\text { LIMK1, } \\
\text { FZD9 }\end{array}$ & & & $7 q 11.2$ & $\begin{array}{l}7 q 11.21 \\
7 q 11.23\end{array}$ \\
\hline $7 q 21.11$ & 76991 & 79296 & 2305 & 6 & & & & & \\
\hline 9p13.3 & 35062 & 35844 & 782 & 31 & & & & & 9p13.3 \\
\hline 10p12.31 & 18796 & 20430 & 1634 & 7 & & & & & \\
\hline $12 q 23.1$ & 94828 & 97459 & 2631 & 18 & & & & & \\
\hline $12 \mathrm{q} 24.31$ & 120738 & 121633 & 895 & 18 & & & & & \\
\hline $13 q$ & & & & Whole q arm & & & & & \\
\hline $13 q 14.2-14.3^{\mathrm{a}}$ & 47839 & 51752 & 3913 & 54 & & & & & $\begin{array}{l}\text { 13q12.11, } \\
13 q 33.3\end{array}$ \\
\hline $13 q 31.1^{\mathrm{a}}$ & 78642 & 80092 & 1450 & 5 & & & & & \\
\hline $13 q 34^{a}$ & 112925 & 113288 & 363 & 8 & $\begin{array}{l}\text { TFDP1, } \\
\text { CUL4A }\end{array}$ & & & & $13 q 34$ \\
\hline $15 q 13.3$ & 29439 & 30347 & 908 & 6 & & & 15q-qter & & \\
\hline $16 q 12.1$ & 48851 & 49785 & 934 & 11 & & & & $16 q$ & \\
\hline $20 \mathrm{p} / \mathrm{q}$ & & & & Whole chr. & & & & $20 \mathrm{p} / \mathrm{q}$ & \\
\hline $20 \mathrm{p} 12.3-\mathrm{p} 12.2^{\mathrm{a}}$ & 7241 & 8681 & 1440 & 6 & & & & & 20q11.1-q12 \\
\hline $\begin{array}{l}\text { 20q11.21- } \\
\text { q11.23 }\end{array}$ & 30417 & 36618 & 6201 & 126 & & & & $20 q 13$ & 20q13.31 \\
\hline $\begin{array}{l}20 q 13.32- \\
q 13.33^{\mathrm{a}}\end{array}$ & 56224 & 62300 & 6076 & 110 & $B R K$ & & & & $21 q 22.3$ \\
\hline $21 \mathrm{q}$ & & & & Whole q arm & & & & & \\
\hline $\begin{array}{l}22 q 12.1 \\
X p / q\end{array}$ & 26643 & 27535 & 892 & $\begin{array}{c}6 \\
\text { Whole chr. }\end{array}$ & & & & $22 q$ & \\
\hline
\end{tabular}

The start and end points of the particular aberration were estimated from CGH Explorer software. Number of genes in the area is according to NCBI database. Previously reported results that match those obtained in this study are also indicated.

${ }^{\mathrm{a}} \mathrm{Gains}$ in at least $30 \%$ of the cases.

alterations that were shared in at least half of these cases were recorded (Figure 3). Loss of Xp21.1 and gains of 20q11.21-q11.23, 13q14.2-q14.3, and $13 q 31.1$ were present in two-thirds of stage 3 cancers. Gains at $5 q 35$ and $13 q$, and losses at 6q16-q22, 2q31.1, and 2q33.3, were present in half of the cases. The long arms of chromosomes 13 and 20 were gained in $50 \%$ of these olfactory neuroblastomas, possibly indicating important roles in tumor progression.

\section{Discussion}

The aim of our study was to identify DNA copy number changes in olfactory neuroblastoma by aCGH. Until recently, genome-wide-profiling studies have relied on conventional CGH in chromosome band resolution. In one study, FISH and single-nucleotide polymorphism microarray analysis were used for one case of olfactory neuroblastoma. ${ }^{3}$ Moreover, the CGH studies of cytogenetic aberrations in olfactory neuroblastoma are few. ${ }^{4-6}$ We performed an oligonucleotide-based aCGH analysis on 13 olfactory neuroblastoma samples, which, to the best of our knowledge, was the first time that array-based CGH was applied to study the copy number changes in this neoplasm. Several copy number changes reported in previous studies were observed in our study, with identical, overlapping, or slightly different minimal common regions of alteration. Gains at the distal parts of 1p, 4, 9p, 13q, $15 q, 22 q$, and $21 q$, and deletions at $4 p$ and $X$, were reported in at least one study. Gains at $7 q 11$ and $20 q$ and deletions at $2 q, 5 q, 6 p, 6 q$, and $18 q$ were detected in two studies (Tables 3 and 4).

Overall, olfactory neuroblastomas have highly complex copy number changes that occur over the entire genome. All samples analyzed showed genomic imbalances with slightly more gains than losses. As expected, aCGH revealed more copy number changes than previous studies that used conventional CGH. Furthermore, our results showed novel aberrations, which were not described in previous 
Table 4 DNA copy number losses in at least $20 \%$ of the cases detected by aCGH

\begin{tabular}{|c|c|c|c|c|c|c|c|c|c|}
\hline $\begin{array}{l}\text { Chromosome } \\
\text { location }\end{array}$ & Start $(k b)$ & Stop $(k b)$ & $\begin{array}{c}\text { Size of area } \\
(k b)\end{array}$ & $\begin{array}{c}\text { No. of genes in } \\
\text { the area }\end{array}$ & $\begin{array}{l}\text { Suggested } \\
\text { target genes }\end{array}$ & $\begin{array}{l}\text { Szymas et al } \\
\text { (1997) }\end{array}$ & $\begin{array}{l}\text { Riazimand } \\
\text { et al (2002) }\end{array}$ & $\begin{array}{l}\text { Bockmühl et } \\
\text { al (2004) }\end{array}$ & $\begin{array}{l}\text { Holland et al } \\
\text { (2007) }\end{array}$ \\
\hline $2 q 31.1$ & 173492 & 175245 & 1753 & 19 & & & & $2 q 22-q 32$ & $2 q 31-q 33$ \\
\hline $2 q 33.3$ & 206359 & 207454 & 1095 & 18 & $A D A M 23$ & & & & \\
\hline $2 q 37.1$ & 231775 & 233528 & 1753 & 38 & & & & & $2 \mathrm{q} 37,2 \mathrm{q} 37.3$ \\
\hline $4 \mathrm{p} 13$ & 41143 & 44546 & 3403 & 19 & & & & $\begin{array}{l}4 \mathrm{p} 13-\mathrm{p} 15 \\
4 \mathrm{p} / \mathrm{q}\end{array}$ & \\
\hline $5 q 31.2$ & 138286 & 138835 & 549 & 11 & & $5 q$ & & $5 q$ & \\
\hline $6 \mathrm{p} 22.1$ & 26803 & 28249 & 1446 & 50 & & & 6p-pter & & \\
\hline $6 \mathrm{p} 21.33$ & 30853 & 31865 & 1012 & 57 & & & & $6 \mathrm{p} 21$ & \\
\hline 6p12.3 & 47054 & 50959 & 3905 & 27 & & & & & \\
\hline $6 q 16.3$ & 101732 & 105348 & 3616 & 2 & & & & $6 q 14-q 23$ & \\
\hline $6 q 21$ & 107952 & 110411 & 2459 & 35 & $\begin{array}{l}\text { FOXO3, } \\
\text { CCNC }\end{array}$ & & & & \\
\hline $\begin{array}{l}6 q 22.1 \\
15 q 11.2-q 24.1\end{array}$ & 113449 & 116631 & $\begin{array}{c}3182 \\
\text { Whole q arm }\end{array}$ & 13 & & & & & $6 q 22-q 24$ \\
\hline $15 \mathrm{q} 13.1^{\mathrm{a}}$ & 27625 & 27834 & 209 & 2 & & & & & \\
\hline $18 q 12.2-q 12.3$ & 31999 & 76014 & 44015 & 197 & & 18 & & $18 q$ & \\
\hline $18 \mathrm{q} 12.2-\mathrm{q} 12.3^{\mathrm{a}}$ & 33268 & 41040 & 7772 & 9 & & & & & \\
\hline $19 q 12$ & 32846 & 34776 & 1930 & 6 & & & & & \\
\hline $19 q 13.11$ & 41195 & 41484 & 289 & 11 & & & & & \\
\hline $19 q 13.32$ & 53405 & 53791 & 386 & 10 & & & & & \\
\hline $19 q 13.43$ & 63057 & 63539 & 482 & 20 & & & & & \\
\hline $22 q 11.23$ & 21670 & 22493 & 823 & 21 & & & & & \\
\hline $22 q 12.1$ & 26506 & 26849 & 343 & 3 & & & & & \\
\hline $\begin{array}{l}22 q 11.1- \\
\text { q11.21 }\end{array}$ & 15393 & 17451 & 2058 & 48 & & & & & \\
\hline $\mathrm{Xp} / \mathrm{q}$ & & & Whole chr. & & & $\mathrm{X}$ & & & \\
\hline
\end{tabular}

The start and end points of the particular aberration were estimated from CGH Explorer software. Number of genes in the area is according to NCBI database. Previously reported results that match the results obtained by this study are also indicated.

${ }^{\mathrm{a}}$ Losses in at least $30 \%$ of the cases.

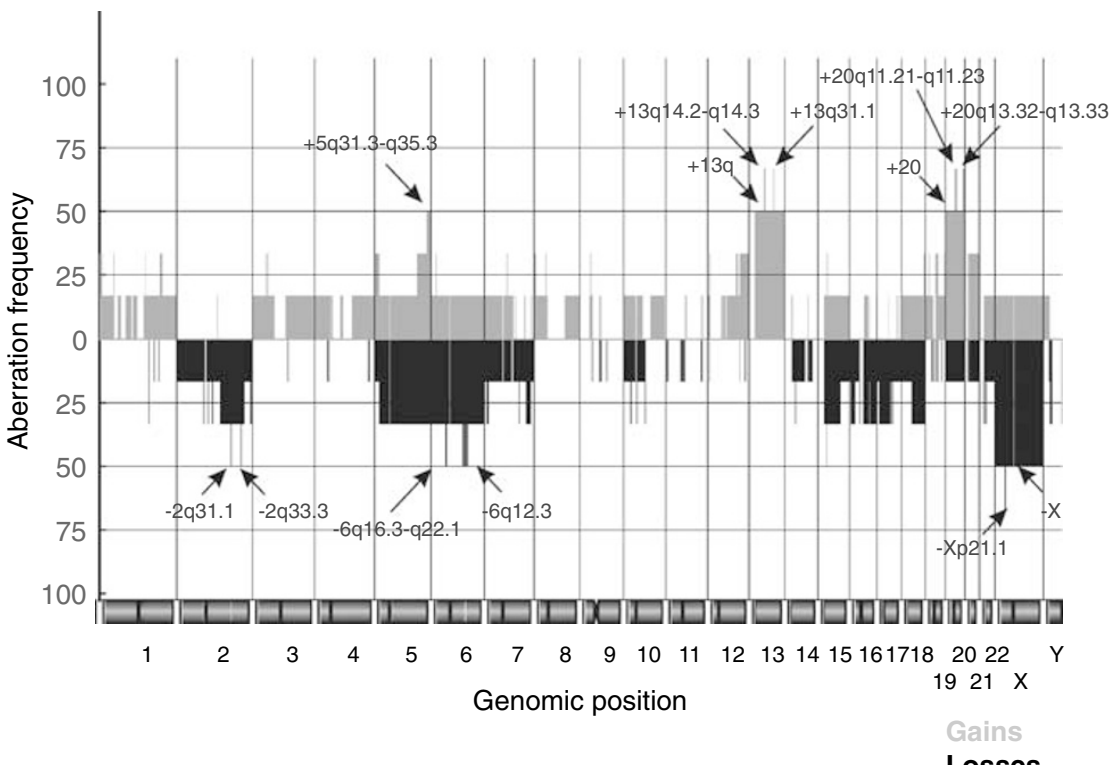

Figure 3 Frequency profile of copy number aberrations in six stage 3 olfactory neuroblastoma samples. The Piecewise Constant Fit algorithm of the CGH Explorer software was applied to identify DNA copy number alterations. Frequencies of copy number gains (gray) and losses (black) are indicated on the $y$ axis. Genomic positions of the aCGH probes are indicated on the $x$ axis. Alterations occurring in at least $50 \%$ of stage 3 cases are marked in the figure.

reports. In accordance with at least two previous studies, we found gains at 7q11.2 and 20q13, and losses at 2q31-q37, 5q, 6p, 6q, and 18q. In addition to these previously reported alterations, we identified novel gains in our samples at 5q34-q35, 6p12.3, 10p12.31, 12q23.1-q24.31, and all of chromosome 
X. Losses at 15q11.2-q24.1, 15q13.1, 19q12-q13, 22q11.1-q11.21, 22q11.23, and 22q12.1 have not been described previously.

We identified a $770 \mathrm{~kb}$ region of chromosomal gain at $7 q 11.2$. This region has been implicated in other cancers, and is overexpressed in prostate carcinomas, adenoid cystic carcinomas, head and neck squamous cell carcinomas, and pancreatic endocrine tumors. ${ }^{9-12}$ Candidate genes located within this region include LIMK1 (NCBI GeneID: 3984), a possible oncogene that contributes to cell cycling and invasion. Another possible candidate is FZD9 (GeneID: 8326), a member of the 'frizzled' gene family that is upregulated in astrocytomas and gastric carcinomas. ${ }^{13,14}$

A $6 \mathrm{Mb}$ region of gain at 20q13.32-q13.33 was also identified. DNA copy number increases at chromosome 20q13 have been observed frequently in a variety of cancers, including breast, ovarian, and squamous cell carcinomas, ${ }^{15}$ suggesting that the region harbors one or more oncogenes. In particular, 20q13.2 has been proposed as a hotspot for candidate genes. ${ }^{15}$ It should also be noted that overexpression at this locus has been associated with reduced patient survival and higher tumor grade. 20q13.3, frequently overexpressed in ovarian cancers, has been recently shown to localize the BRK tyrosine kinase gene (GeneID: 5753), which is thought to have an important role in the development of ovarian cancers. ${ }^{16}$

Losses occurring at chromosome $2 \mathrm{q}$ have been described for various carcinomas, including head and neck squamous cell carcinoma, ${ }^{17-19}$ breast carcinoma, ${ }^{20}$ lung carcinoma,${ }^{21}$ neuroblastoma, ${ }^{22}$ cervical cancer, ${ }^{23}$ and prostate adenocarcinoma. ${ }^{24}$ Studies using different approaches have increasingly shown that the most affected region is 2q32-q37. This region also seems to be implicated in the development of ONB, as it has been reported in three cytogenetic studies including our investigation. Several candidate tumor suppressor genes have been suggested, including ADAM23 (GeneID: 8745), thought to function as an adhesion molecule, which promotes the attachment of neural cells. ${ }^{25}$ This gene at $2 \mathrm{q} 33.3$ was lost in $20 \%$ of all olfactory neuroblastoma samples analyzed and, more significantly, in $50 \%$ of stage 3 tumors. Takada et al have suggested an essential role for this candidate tumor suppressor gene in the progression of gastric cancer, and our results indicate that this might be the case for olfactory neuroblastoma as well.

Another area of loss identified in our study, and also reported by Bockmühl et $a l^{4}$ and Holland et $a l^{3}{ }^{3}$ is located at 6q21-22. This region is frequently deleted in a variety of neoplasms, including pancreatic endocrine tumors, ${ }^{26}$ prostate carcinoma, ${ }^{27}$ breast carcinoma, ${ }^{28}$ and central nervous system lymphomas. ${ }^{29}$ Three main putative tumor suppressor genes have been proposed, including FOXO3 (GeneID: 2309), CCNC (GeneID: 892), and PTPRK
(GeneID: 5796). The first two candidate genes are in the regions found to be deleted in our samples.

Our study also identified two small gains at 9p13.3 (782 kb) and 13q34 (363 kb) that were previously reported by Holland et al. The 9p13.3 locus has been shown to be gained in prostate cancer cell lines in two recent studies using aCGH. ${ }^{30,31}$ These authors suggested the involvement of three genes, including UBE2R2 (GeneID: 54926), DCTN3 (GeneID: 11258), and IL-11RA (GeneID: 3590). Kamradt et al screened 20 primary prostate cancer samples and found that only $I L-11 R A$ was gained in $75 \%$ of the tumors, whereas only DCTN3 was not gained in any of the cases; both genes were gained together in $10 \%$ of the tumors. This observation led the authors to suggest that $I L-11 R A$ is the target of gain rather than DCTN3. Gains at 13q34 have also been described previously in different cancers, including breast cancer, hepatocellular carcinoma, esophageal squamous cell carcinoma, and lung adenocarcinoma. ${ }^{32}$ The TFDP1 (GeneID: 7027) and CUL4A (GeneID: 8451) genes were suggested as target genes that possibly have a role in carcinogenesis. Further studies are required to determine if these genes are involved in olfactory neuroblastoma.

As for most tumors, stage is the most important parameter associated with survival in olfactory neuroblastoma. ${ }^{2}$ Our results clearly indicate that alterations in $20 \mathrm{q}$ and $13 \mathrm{q}$ are important in the progression of olfactory neuroblastoma. Gain of $20 \mathrm{q}$ has been widely associated with progression of several tumors, including breast carcinoma, ${ }^{33}$ cervical carcinoma, ${ }^{34}$ and pancreatic carcinoma. ${ }^{35}$ Both losses and gains of chromosome 13q have been noted in many recent studies of various tumors, suggesting the existence of novel oncogenes or tumor suppressor genes or both in this region. Furthermore, this region has been reported to contain microRNAs that could function as tumor suppressor genes or oncogenes. ${ }^{36}$ The only known tumor-related gene in this region is the tumor suppressor gene $R B 1$. It is associated with loss of function or decreased gene expression in tumor cells and, consequently, cannot be considered further. Lillington et $a l^{37}$ found that gain of $13 \mathrm{q}$ is more frequent in older patients, whose tumors display more abnormalities than those in younger patients. These authors have also reported that gain of $13 q$ is seen more frequently in poorly differentiated malignancies, which typically contain more abnormalities than well-differentiated cancers. Gains of both $13 q$ and $20 \mathrm{q}$ are seen in colorectal carcinomas and their progression. ${ }^{38,39}$

In conclusion, we have identified novel chromosomal regions, which are frequently altered in olfactory neuroblastoma. When the results of our study are combined with previously published data, several regions are identified that are consistently abnormal in olfactory neuroblastoma. These regions are also frequently associated with tumorigenesis of a variety of cancers, especially carcinomas. A 
number of genes located at these sites have been suggested to be important, but further experiments are required to determine their roles in olfactory neuroblastoma.

\section{Disclosure}

The authors state no conflict of interest.

\section{References}

1 Barnes L, (ed) Pathology and Genetics of Head and Neck Tumours. International Agency for Research on Cancer (IARC), Lyon, France, 2005, pp 430.

2 Morita A, Ebersold MJ, Olsen KD, et al. Esthesioneuroblastoma: prognosis and management. Neurosurgery 1993;32:706-714, discussion 714-715.

3 Holland H, Koschny R, Krupp W, et al. Comprehensive cytogenetic characterization of an esthesioneuroblastoma. Cancer Genet Cytogenet 2007;173:89-96.

4 Bockmuhl U, You X, Pacyna-Gengelbach M, et al. CGH pattern of esthesioneuroblastoma and their metastases. Brain Pathol 2004;14:158-163.

5 Riazimand SH, Brieger J, Jacob R, et al. Analysis of cytogenetic aberrations in esthesioneuroblastomas by comparative genomic hybridization. Cancer Genet Cytogenet 2002;136:53-57.

6 Szymas J, Wolf G, Kowalczyk D, et al. Olfactory neuroblastoma: detection of genomic imbalances by comparative genomic hybridization. Acta Neurochir (Wien) 1997;139:839-844.

7 Mills SE, Frierson Jr HF. Olfactory neuroblastoma. A clinicopathologic study of 21 cases. Am J Surg Pathol 1985;9:317-327.

8 Lingjaerde OC, Baumbusch LO, Liestol K, et al. CGHexplorer: a program for analysis of array-CGH data. Bioinformatics 2005;21:821-822.

9 Davila M, Frost AR, Grizzle WE, et al. LIM kinase 1 is essential for the invasive growth of prostate epithelial cells: implications in prostate cancer. J Biol Chem 2003;278:36868-36875.

10 Kasamatsu A, Endo Y, Uzawa K, et al. Identification of candidate genes associated with salivary adenoid cystic carcinomas using combined comparative genomic hybridization and oligonucleotide microarray analyses. Int J Biochem Cell Biol 2005;37:1869-1880.

11 Bockmuhl U, Schluns K, Schmidt S, et al. Chromosomal alterations during metastasis formation of head and neck squamous cell carcinoma. Genes Chromosomes Cancer 2002;33:29-35.

12 Stumpf E, Aalto Y, Hoog A, et al. Chromosomal alterations in human pancreatic endocrine tumors. Genes Chromosomes Cancer 2000;29:83-87.

13 Zhang Z, Schittenhelm J, Guo K, et al. Upregulation of frizzled 9 in astrocytomas. Neuropathol Appl Neurobiol 2006;32:615-624.

14 Kirikoshi H, Sekihara H, Katoh M. Expression profiles of 10 members of frizzled gene family in human gastric cancer. Int J Oncol 2001;19:767-771.

15 Quinlan KG, Verger A, Yaswen P, et al. Amplification of zinc finger gene 217 (ZNF217) and cancer: when good fingers go bad. Biochim Biophys Acta 2007;1775:333-340.

16 Schmandt RE, Bennett M, Clifford S, et al. The BRK tyrosine kinase is expressed in high-grade serous carcinoma of the ovary. Cancer Biol Ther 2006;5:1136-1141.

17 Beder LB, Gunduz M, Ouchida M, et al. Genome-wide analyses on loss of heterozygosity in head and neck squamous cell carcinomas. Lab Invest 2003;83:99-105.

18 Yamamoto N, Mizoe J, Numasawa H, et al. Allelic loss on chromosomes 2q, 3p and 21q: possibly a poor prognostic factor in oral squamous cell carcinoma. Oral Oncol 2003;39:796-805.

19 Cengiz B, Gunduz M, Nagatsuka H, et al. Fine deletion mapping of chromosome 2q21-37 shows three preferentially deleted regions in oral cancer. Oral Oncol 2007;43:241-247.

20 Piao Z, Lee KS, Kim H, et al. Identification of novel deletion regions on chromosome arms $2 q$ and $6 p$ in breast carcinomas by amplotype analysis. Genes Chromosomes Cancer 2001;30:113-122.

21 Otsuka T, Kohno T, Mori M, et al. Deletion mapping of chromosome 2 in human lung carcinoma. Genes Chromosomes Cancer 1996;16:113-119.

22 Takita J, Yang HW, Chen YY, et al. Allelic imbalance on chromosome $2 q$ and alterations of the caspase 8 gene in neuroblastoma. Oncogene 2001;20:4424-4432.

23 Narayan G, Pulido HA, Koul S, et al. Genetic analysis identifies putative tumor suppressor sites at 2q35q36.1 and 2q36.3-q37.1 involved in cervical cancer progression. Oncogene 2003;22:3489-3499.

24 Ueda T, Komiya A, Suzuki H, et al. Loss of heterozygosity on chromosome 2 in japanese patients with prostate cancer. Prostate 2005;64:265-271.

25 Takada H, Imoto I, Tsuda H, et al. ADAM23, a possible tumor suppressor gene, is frequently silenced in gastric cancers by homozygous deletion or aberrant promoter hypermethylation. Oncogene 2005;24: 8051-8060.

26 Barghorn A, Speel EJ, Farspour B, et al. Putative tumor suppressor loci at 6q22 and 6q23-q24 are involved in the malignant progression of sporadic endocrine pancreatic tumors. Am J Pathol 2001;158: 1903-1911.

27 Kim JH, Dhanasekaran SM, Mehra R, et al. Integrative analysis of genomic aberrations associated with prostate cancer progression. Cancer Res 2007;67: 8229-8239.

28 Theile M, Seitz S, Arnold W, et al. A defined chromosome $6 \mathrm{q}$ fragment (at D6S310) harbors a putative tumor suppressor gene for breast cancer. Oncogene 1996;13:677-685.

29 Nakamura M, Kishi M, Sakaki T, et al. Novel tumor suppressor loci on 6q22-23 in primary central nervous system lymphomas. Cancer Res 2003;63:737-741.

30 Saramaki OR, Porkka KP, Vessella RL, et al. Genetic aberrations in prostate cancer by microarray analysis. Int J Cancer 2006;119:1322-1329.

31 Kamradt J, Jung V, Wahrheit K, et al. Detection of novel amplicons in prostate cancer by comprehensive genomic profiling of prostate cancer cell lines using oligonucleotide-based arrayCGH. PLoS ONE 2007; 2:e769.

3232 Abba MC, Fabris VT, Hu Y, et al. Identification of novel amplification gene targets in mouse and human breast cancer at a syntenic cluster mapping to mouse ch8A1 and human ch13q34. Cancer Res 2007;67: 4104-4112.

33 Hodgson JG, Chin K, Collins C, et al. Genome amplification of chromosome 20 in breast cancer. Breast Cancer Res Treat 2003;78:337-345. 
34 Wilting SM, Snijders PJ, Meijer GA, et al. Increased gene copy numbers at chromosome $20 \mathrm{q}$ are frequent in both squamous cell carcinomas and adenocarcinomas of the cervix. J Pathol 2006;209: 220-230.

35 Loukopoulos P, Shibata T, Katoh H, et al. Genomewide array-based comparative genomic hybridization analysis of pancreatic adenocarcinoma: identification of genetic indicators that predict patient outcome. Cancer Sci 2007;98:392-400.

36 Calin GA, Croce CM. MicroRNAs and chromosomal abnormalities in cancer cells. Oncogene 2006;25: 6202-6210.

37 Lillington DM, Kingston JE, Coen PG, et al. Comparative genomic hybridization of 49 primary retinoblastoma tumors identifies chromosomal regions associated with histopathology, progression, and patient outcome. Genes Chromosomes Cancer 2003;36:121-128.

38 Lips EH, de Graaf EJ, Tollenaar RA, et al. Single nucleotide polymorphism array analysis of chromosomal instability patterns discriminates rectal adenomas from carcinomas. J Pathol 2007;212:269-277.

39 Hermsen M, Postma C, Baak J, et al. Colorectal adenoma to carcinoma progression follows multiple pathways of chromosomal instability. Gastroenterology 2002;123:1109-1119. 